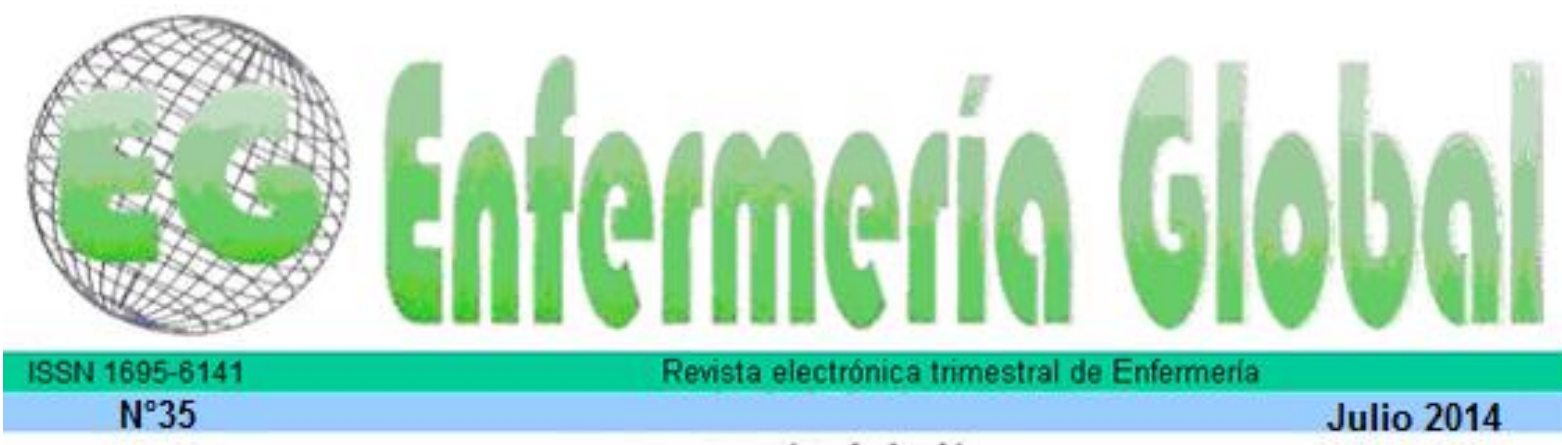

www.um.es/egloball

\title{
REVISIONES
}

\section{La cronicidad de la fibromialgia. Una revisión de la literatura}

The chronic nature of the fibromyalgia. A literature review

\section{*Triviño Martínez, Ángeles **Solano Ruiz, Mª Carmen ***Siles González, José}

\begin{abstract}
*Estudiante de Doctorado en Ciencias de la Salud, Universidad de Alicante. Enfermera colaboradora en la Asociación de pacientes de fibromialgia (AFEFE). España. E-maill: angelatrivi1@hotmail.com **Enfermera. Doctora en Antropología. Profesora Titular en la Facultad de Ciencias de la Salud, Universidad de Alicante. ***Enfermero. Doctor en Historia. Profesor Titular en la Facultad de Ciencias de la Salud, Universidad de Alicante. España.
\end{abstract}

Palabras clave: fibromialgia; dolor; vivencia; investigación cualitativa y fenomenología. Keywords fibromyalgia; pain; experience; qualitative research and phenomenology.

\section{RESUMEN}

La fibromialgia (FM) se caracteriza por ser una enfermedad crónica de etiología desconocida con múltiples consecuencias tanto para la persona que la padece como para su familia y su propio entorno social y laboral.

El objetivo de este trabajo se centra en recopilar las experiencias y vivencias de las personas que padecen fibromialgia.

Para ello se ha realizado una revisión bibliográfica donde se han seleccionado artículos con una metodología de carácter cualitativo publicados durante el período 2005 a 2012 en las siguientes bases de datos: Pubmed, IME, CUIDEN y CINAHL. Igualmente en las revistas Scielo, Index de enfermería, Journal Advanced Nursing y Journal Clinical Nursing.

Tras la revisión bibliográfica 42 artículos fueron seleccionados por cumplir los criterios de inclusión donde se identifican los siguientes temas claves: diagnóstico de la enfermedad, tratamiento, la enfermedad y el dolor, alteración del sueño, la fatiga, deterioro cognitivo, impacto laboral, impacto emocional, necesidades y demandas de los pacientes hacia los profesionales sanitarios, vivencia de la enfermedad y búsqueda de ayuda e información.

Los pacientes no son escuchados en el principio de su enfermedad, son considerados enfermos con trastornos psicológicos lo que les ocasiona incertidumbre y una estigmatización social. El punto de 
inflexión es recibir un diagnóstico firme, a partir de aquí la persona podrá elaborar estrategias de afrontamiento durante su proceso. Los síntomas más importantes asociados a la enfermedad son el dolor, la fatiga, la alteración del sueño y el deterioro cognitivo que repercutirán en sus actividades a nivel social y laboral.

\section{ABSTRACT}

Fibromyalgia (FM) is characterized as a chronic disease of unknown etiology with multiple consequences for the person who suffers it, his family and own social-working environment.

The aim of this paper focuses on collecting the experiences of people with fibromyalgia. In order to do so, it was carried out a bibliographic review in which the articles were selected by qualitative methodology. The articles were published during the period 2005-2012 in the following databases: PubMed, IME, CUIDEN and CINAHL. Also, in Scielo Journals, Nursing Index, Journal Advanced Nursing and Clinical Nursing Journal.

Following the literature review, 42 items met the inclusion criteria and were bracketed on the following category: disease diagnosis, treatment, disease and pain, sleep disturbance, fatigue, cognitive impairment, labor impact, emotional impact, needs and demands of patients to health professionals, experience of illness and seeking help and information.

The patients' voice is not heard or taken into consideration at the beginning of their illness. They are considered patients with psychological disorders which cause them uncertainty and social stigmatization. The inflexion point is getting a firm diagnosis; from this point the person will be able to create strategies during their own process. The most significant symptoms associated with the disease are: pain, fatigue, sleep disturbance and cognitive impairment that will affect their social and labor activities.

\section{INTRODUCCIÓN}

Las enfermedades crónicas como es el caso de la fibromialgia (FM) representan hoy día uno de los mayores retos planteados a la sanidad, tanto por su elevada prevalencia como por las consecuencias que de ella se derivan al paciente, a sus familiares y al entorno social y laboral ${ }^{(1),(2)}$.

La enfermedad de fibromialgia ocasiona un elevado consumo de recursos asistenciales además de importante coste personal, social y laboral ${ }^{(2.3)}$. Se estima que esta enfermedad afecta entre el 2 y el 2,7 \% de la población española mayor de 20 años, siendo mucho más elevada para las mujeres: el 4,2 \% en contraposición del 0,2 \% para varones. Cada año se diagnostican en España 120.00 casos de FM y se estima que existen casi dos millones de afectados ${ }^{(1),(2),(3)}$.

La fibromialgia (FM) es una enfermedad de etiología desconocida caracterizada por la presencia de dolor localizado por lo general en zonas musculares, tendinosas, articulares y viscerales ${ }^{(4,5)}$. Aparecen además, otros síntomas como fatiga, depresión, ansiedad, rigidez articular, cefalea, sensación de tumefación en manos, trastornos del sueño y parestesias en extremidades; igualmente, hipersensibilidad en múltiples puntos predefinidos denominados puntos gatillo o tigger points, los cuales corresponden a zonas fisiológicamente más sensibles a la presión. A pesar del dolor, las estructuras afectadas no presentan daño o inflamación ${ }^{(4),(5),(6),(7)}$.

En la mayoría de los casos de FM descritos empiezan después de hechos puntuales, infecciones víricas o bacterianas, accidentes de automóvil, separación matrimonial, problemas familiares, etc ${ }^{(2,5,6)}$. Otras veces aparece después de sufrir enfermedades que debilitan el organismo (artritis reumatoide, lupus, etc) considerándose estas 
circunstancias como desencadenantes pero no causantes ${ }^{(6,7,8)}$. En la actualidad, son muchos los trabajos de investigación dedicados a conocer las causas de esta enfermedad pero no existe acuerdo en que la FM esté provocada por factores orgánicos o trastornos psicológicos ${ }^{(1),(2),(3),(4),(5),(6)}$.

El desconocimiento de su etiología favorece el peregrinaje de las personas que la padecen a distintos especialistas previamente al diagnóstico (digestivo, corazón, traumatología, psiquiatría, reumatología, rehabilitación...) llegando a consultar hasta 15 especialistas antes de llegar al diagnóstico definitivo ${ }^{(2,4,7)}$.

Desde hace 30 años se acepta el modelo biopsicosocial de la enfermedad, según éste tanto el dolor como la fatiga son la consecuencia de diferentes factores (trauma, enfermedad, procedimientos médicos, defectos congénitos, etc) ${ }^{(5,7,9)}$. El modelo biomédico imperante etiqueta como alteración psicológica todo aquello donde se desconoce la causa que lo produce ${ }^{(7,9)}$. En este sentido Hidalgo plantea que la ausencia de daño orgánico no significa que los signos y síntomas sean psicógenos.

Este concepto sería la base de un tratamiento integrado en el que se unifique elementos como: coordinación de educación, el ejercicio aeróbico y la terapia cognitivo-conductual que demuestran ser efectivos en el alivio de los síntomas de la enfermedad ${ }^{(4,9,10)}$.

La fibromialgia afecta a las esferas biológica, psicológica y social de las personas que la padecen, comprometiendo su calidad de vida y repercutiendo en la capacidad física, actividad intelectual, estado emocional, relaciones personales y trabajo, además de importantes repercusiones económicas que de ella se derivan producidas por la discapacidad laboral que ocasiona a la persona además de un importante gasto sanitario $^{(6,8,10,11)}$

El objetivo de este trabajo es recoger las percepciones, vivencias y necesidades de las personas que padecen fibromialgia a través de una revisión de estudios de diseño cualitativo.

\section{MATERIAL Y MÉTODOS}

Se llevó a cabo una revisión bibliográfica de la producción científica relacionada con la fibromialgia entre los años 2005 y 2012. Las bases de datos consultadas han sido PUBMED, IME, CINAHL y CUIDEN. Así mismo se han consultado revistas de impacto como Scielo, Journal Advanced Nursing, Index de Enfermería y Journal Clinical Nursing. Esta búsqueda se ha realizado entre los meses de mayo a julio de 2012.

La estrategia de búsqueda se ha conducido desde los aspectos generales de la enfermedad utilizando las palabras claves dolor y fibromialgia cuyo resultado fue una multitud de artículos 7.118 centrados en la fisiopatología, los criterios diagnósticos de la enfermedad y tratamientos farmacológicos lo que obligó a restringir la búsqueda añadiendo los descriptores investigación cualitativa, vivencia y fenomenología para a continuación seleccionar los artículos más relevantes en el campo de la investigación cualitativa, en primer lugar se seleccionaron 68. Todos los estudios fueron detenidamente leídos, se procedió a la extracción de la información y datos relevantes asociados al objetivo de este artículo lo que obtuvo un resultado de inclusión de 42 artículos. 
Hay un total de 306 revistas diferentes que publican sobre esta área. EL área temática más trabajada ha sido el estudio de los potenciales mecanismos patofisiológicos $(31,01 \%)$, con incremento de artículos vinculados con aspectos psicológicos en los últimos años ${ }^{(10,12)}$.

Las revistas que aportan un mayor número de artículos son aquellas especializadas en la enfermedad a estudio pero centradas en su fisiopatología y últimos tratamientos aplicados como son Revistas de Reumatología, Revistas de Psicología o Revista especializadas en el dolor; la mayoría de los trabajos se realizan en Estados Unidos, Canadá y Alemania. Otras revistas consultados como Scielo, Index de Enfermería, Journal of Advanced Nursing y Journal Clinical Nurssing aportan más cantidad de artículos en los que se emplea la metodología cualitativa, incluyendo historias de vida y relatos biográficos en los que la persona es la protagonista contando la experiencia subjetiva de su enfermedad.

El incremento de investigaciones y artículos publicados sobre el tema en el período de 1996 a 2005 se triplicaron con respecto a la década anterior. La mayor parte de estos artículos tratan sobre fisiopatología, criterios diagnósticos y de clasificación y sobre evidencias de tratamiento ${ }^{(1,12)}$.

En cuanto a la selección de los artículos se han elegido aquellos que tienen una metodología cualitativa donde se explora la vivencia de la patología por parte de la persona en su vida cotidiana para comprender con naturalidad el fenómeno desde una mirada profunda y el significado que se le atribuye.

Criterios de inclusión:

-Artículos originales en bases de datos y revistas científicas de investigación primaria publicados en inglés o en castellano entre los años 2005 y 2012.

-Todos aquellos textos de método cualitativo donde se explora la vivencia de las personas ante la FM desde que son diagnosticadas.

-Los estudios cualitativos en el que el método de recolección de datos fueran entrevistas individuales a los participantes donde se permite presentar sus percepciones con sus propias palabras, es decir, las entrevistas no estructuradas o semi-estructuradas.

-Textos en que es la propia persona la que cuenta su experiencia ante la enfermedad como son las historias de vida o relatos biográficos.

\section{RESULTADOS}

En la presente revisión de la literatura se identifican los siguientes temas principales donde los pacientes describen dominios de síntomas que interfieren con su función y calidad de vida (Tabla I), destacando:

1. Diagnóstico de la enfermedad.

2. Tratamiento.

3. La enfermedad y el dolor.

4. Alteración del sueño. 

5. La fatiga.
6. Deterioro cognitivo.
7. Impacto laboral.
8. Impacto emocional.
9. Necesidades y demandas de los pacientes hacia los profesionales sanitarios.
10. La vivencia de la enfermedad.
11.Búsqueda de ayuda e información.

\section{Temas Clave}

\section{- Diagnóstico de la enfermedad}

La principal dificultad para diagnosticar esta patología es que se desconoce su causa y que no existen alteraciones que se puedan detectar fácilmente para llegar a un diagnóstico rápido y certero ${ }^{(1,2,3,5,7) \text {, }}$

El paciente tiene que presentar dolores generalizados en los 4 cuadrantes del cuerpo más la espalda, por un período de al menos 3 meses y debe tener dolor en 11 de los 18 puntos sensibles establecidos para su diagnóstico ${ }^{(6,8,13)}$.

La FM con frecuencia coexiste con otras condiciones médicas y es importante reconocer y tratar la fibromialgia como un trastorno diferente ${ }^{(5,6,10)}$. Algunos médicos pueden no reconocer esta enfermedad o pueden ver el estado como un trastorno psiquiátrico o simplemente no creíble; como resultado los pacientes se pueden sentir confundidos, frustrados y con una baja autoestima debido principalmente a su peregrinaje hasta el diagnóstico ${ }^{(4,9,13,14)}$.

Las personas enfermas relatan una vida antes y después del diagnóstico ya que repercute en que no son creídas por sus familiares y amigos ya que la invisibilidad de la fibromialgia es una dificultad a la que se enfrentan la mayoría de los pacientes $(4,11,13,14,15)$

\section{- Tratamiento}

Desde el punto de vista del planteamiento terapéutico no se conoce ningún tratamiento con capacidad para curarla, su objetivo es aliviar los síntomas y la adaptación del enfermo ya que pocas son las medidas farmacológicas de demostrada evidencia exceptuando los beneficios de antiinflamatorios, analgésicos reguladores del sueño particularmente de antidepresivos o relajantes musculares y las terapias conductuales-cognitivas ${ }^{(10,16,17)}$

Es por ello que cada vez con más preponderancia tienen protagonismo las intervenciones no farmacológicas en este colectivo, los estudios mencionan otras terapias alternativas que incluyen: la acupuntura, el biofeedback, modificaciones de la dieta/ejercicio, el calor, hidroterapia, masaje, taichí y la meditación o yoga ${ }^{(10,16,17,18)}$.

\section{- La enfermedad y el dolor}

El dolor es el principal de los problemas de estos pacientes que lo describen como incapacitante para la actividad diaria pero no para la movilidad, es resistente a los diagnósticos y a los tratamientos convencionales ${ }^{(11,19,20,21)}$ 
Para poder convivir con un dolor crónico en los últimos 10 años los modelos cognitivoconductuales de manejo del dolor han ido más allá del enfoque tradicional sobre las estrategias de afrontamiento y el control percibido sobre el dolor, para incorporar la atención y los enfoques basados en la aceptación del dolor que se asocia con un mejor funcionamiento físico, emocional y social ${ }^{(4,10,19,20,21,22)}$.

El punto de inflexión más significativo en el proceso de aceptación es recibir el diagnóstico, información, el apoyo social percibido de la familia, amigos y profesionales de la salud $^{(11,20,23,24,25)}$.

\section{Alteración del sueño}

Una repercusión importante en la calidad de vida de estos pacientes son las alteraciones del sueño, desean una mejora en su capacidad para dormir indicando que tanto la fatiga y el dolor se relacionan directamente con la calidad del sueño $(26,27,28,29,30)$.

Un estudio realizado por Martín et ${ }^{(29)}$ al tuvo como objetivo evaluar el impacto de la calidad del sueño en pacientes con FM a través de una investigación cualitativa donde se utilizó la entrevista semi-estructurada donde se demuestra el negativo impacto que tiene en la vida de los pacientes aumentando los niveles de dolor y la disminución de los niveles de funcionamiento físico incluyendo su estado de ánimo, las relaciones familiares y sociales y la productividad en el trabajo. Los participantes informaron que desde que padecen FM ya no son capaces de experimentar una noche de sueño realmente reparador que da lugar a una sensación restaurada o descansada al despertar indicando que la calidad del sueño asociado al tratamiento es de importancia crítica.

\section{La fatiga}

Los pacientes describen la fatiga como sensación de debilidad o pesadez, con dificultad para sentirse motivado en realizar sus tareas, tener que hacer las cosas más lentamente, dificultad para concentrarse, pensar o recordar las cosas, abrumadora sensación de cansancio que no se alivia con el descanso o dormir y no es proporcional al esfuerzo realizado ${ }^{(20,26,27,28)}$.

Se identifica la fatiga como otro de los peores síntomas asociados con la fibromialgia junto con el dolor, es una presencia constante en las vidas de los pacientes que a menudo tienen que medir sus pasos para asegurarse de que las tareas previstas durante el día las pueden llevar a cabo ${ }^{(10,11,19,26,28,31)}$.

Humphrey et al ${ }^{(31)}$ realizó un estudio con el fin de entender mejor los aspectos de la fatiga que podrían ser únicos para la FM así como el impacto que tiene en la vida de los pacientes que la describieron un cansancio más intenso, constante y persistente que no se alivia con el descanso y no es proporcional al esfuerzo realizado, interfiere en las actividades diarias y hace que sea difícil concentrarse.

\section{Deterioro cognitivo}

La FM afecta la cognición de los pacientes en particular su memoria y los procesos de pensamiento como consecuencia no son capaces de operar en los mismos niveles de agudeza mental que los que tenían antes de la aparición de la enfermedad, olvidando 
tareas importantes y a menudo incapaces de expresar sus pensamientos a los demás por lo que afecta a su capacidad de concentrarse y expresarse con claridad $(10,26,28$, 32,33)

\section{Impacto laboral}

La limitada capacidad física, una mayor necesidad de descanso, la incapacidad para realizar tareas repetitivas o la incapacidad para concentrarse relacionado con el deterioro cognitivo influyen en pacientes con FM para responder a las demandas físicas, psicosociales y de organización en el trabajo ${ }^{(20,26,28)}$.

Las largas jornadas de trabajo, trabajo estático prolongado sobre todo si se tienen que utilizar los brazos, marcha larga y levantar o cargar objetos pesados dan lugar a un empeoramiento de los síntomas como son dolor, agotamiento y fatiga ${ }^{(23,25,31)}$.

También se perciben como altamente exigentes los trabajos en los que se utilicen instrumentos manuales así como los que demanden un poder de concentración mayor o donde haya una baja temperatura al aire libre o un nivel de sonido alto de la maquinaria $^{(34,35)}$

Informan de que tienen que cambiar con frecuencia de trabajo o tener una reducción de horas lo que repercute en su situación económica ${ }^{(10,34,35)}$.

\section{Impacto emocional}

A nivel psicológico existe una elevada evidencia científica que relaciona la FM con distintos problemas anímicos, siendo de mayor prevalencia la ansiedad y la depresión en un $68 \%$ de los casos; su aparición cronifica, empeora el curso y la evolución de la enfermedad ${ }^{(33,36)}$.

Entre las causas que se barajan destacan: el mecanismo de estrés crónico, el dolor, el retraso diagnóstico, la falta de eficacia de los tratamientos, los problemas laborales y el entorno socio-familiar ${ }^{(27,32,33)}$.

Los estudios identifican sentimientos tales como la frustración, vergüenza, culpa y aislamiento en los pacientes con respecto a su incapacidad para ser entendido por la comunidad médica y por quienes le rodean, así como la frustración con ellos mismos por la pérdida de habilidades cognitivas o pérdida de la identidad sexual ${ }^{(11,20,28)}$.

El desconocimiento sobre su problema y posibles soluciones, la incertidumbre y la incomprensión contribuyen a sumirlas en un estado de depresión y actitud negativa, pero este círculo vicioso se rompe al recibir el diagnóstico que les ayuda a asumir la enfermedad, a aprender a vivir con ella y a buscar estrategias de afrontamiento como terapias alternativas e información ${ }^{(20,25,28,31,32,36)}$.

\section{Necesidades y demandas de los pacientes hacia los profesionales}

Los pacientes demandan a los profesionales sanitarios interés, comprensión, apoyo, empatía y capacidad de comunicación, así como conocimientos sobre la enfermedad y habilidades para transmitirlos e informarles durante todo el proceso y del intercambio de puntos de vista ${ }^{(1,11,26,37,38)}$. 
Esperan también que sus profesionales puedan dedicarles el tiempo suficiente en la consulta, solicitan información sobre lo que pueden esperar de su enfermedad y su proceso, sobre su pronóstico y sobre terapias y cuidados ${ }^{(4,10,20,38,39)}$.

Los pacientes perciben que los profesionales sanitarios tienen poco tiempo y por ello no pueden escuchar sus quejas, limitándose a la prescripción de fármacos lo que redunda en una falta de respuesta a sus necesidades ${ }^{(6,8,15,16,38,39)}$.

Las intervenciones educativas y actividades orientadas al auto-cuidado mejoran la calidad de vida de los pacientes con fibromialgia, los ayudan a conocerse mejor a sí mismos y a desarrollar ciertas capacidades necesarias para el afrontamiento de la enfermedad, como la de elegir, la de actuar por sí mismos o la de negociar $(4,18,25,40)$; los profesionales sanitarios deben comprender la importancia de la esperanza en la experiencia de una enfermedad crónica ya que tiene un poder significativo en ayudar a las personas a entender su potencial durante todo su proceso ${ }^{(38,40,41)}$.

\section{Vivencia de la enfermedad}

Desde que un paciente inicia su contacto con la medicina oficial hasta el diagnóstico transcurre una meda de tiempo de unos 7 años; durante ese tiempo los enfermos se encuentran en una situación de incertidumbre puesto que ni ellos ni su entorno social y familiar consideran que el dolor sea una razón legítima para interrumpir las actividades cotidianas ${ }^{(1,7.10,26,28,39)}$.

Las personas no son tomadas en serio por sus amigos, familiares y profesionales de la salud, ya que parecen sanas y la percepción que los pacientes tienen de su enfermedad es de un origen somático ${ }^{(5,8,25,28)}$ debido a su largo itinerario terapéutico desarrollan estrategias narrativas de su padecimiento -como la forma de expresión o la ocultación de sus síntomas- que les permitan no ser consideradas como enfermos psiquiátricos o como simuladores de sus síntomas ${ }^{(4,10,13,19,31)}$.

El estudio de Wuytack et al ${ }^{(26)}$ reveló que la FM impregna todos los aspectos de la vida, los participantes resaltaban que habían dejado de "funcionar" dando lugar a sentimientos de inutilidad y pérdida de la identidad, las actividades de ocio también se vieron muy afectadas así como la alteración de los lazos familiares algunos de los cuales fueron reforzados, sin embargo otros se quebraron.

La frustración derivada de la incomprensión domina en todos ellos por lo que prefieren no compartir sus experiencias con personas que no padezcan su enfermedad $(5,10,14,26,37)$

Los pacientes informan trastornos en las relaciones con familiares y amigos, aislamiento social, reducción de las actividades de la vida diaria y de ocio, evitan la actividad física y la pérdida de la carrera profesional ${ }^{(17,20,32,42)}$

Desean establecer un equilibrio entre el deseo de permanecer independientes el mayor tiempo posible y la necesidad de apoyo por parte de los miembros de la familia y amigos $(19,23,28,36,39,41)$. 


\section{Las asociaciones de pacientes}

Los pacientes acuden a las asociaciones de pacientes con fibromialgia en busca de refugios seguros donde compartir sus sentimientos con otras personas en condiciones similares, ya que les proporcionan información apoyo y una salida para la ira y la frustración ${ }^{(4,25,42)}$.

Asociarse es una estrategia que utilizan los pacientes, como consecuencia muestran una mejor actitud ante la enfermedad, están más informadas, se sienten seguras y parece que viven la enfermedad de forma más positiva que quienes no frecuentan alguna asociación de pacientes; explican estos beneficios positivos por el apoyo de otras pacientes asociadas y los servicios específicos que les ofrece la asociación $(1,4,36,39,42)$

Tabla I: Características de los principales estudios incluidos en la revisión bibliográfica.

\begin{tabular}{|c|c|c|c|c|c|c|}
\hline $\begin{array}{l}\text { Autores } \\
\text { Fecha de } \\
\text { Publicación } \\
\text { País }\end{array}$ & $\begin{array}{l}\text { Diseño del } \\
\text { estudio }\end{array}$ & $\begin{array}{l}\text { Sujetos } \\
\text { participanes }\end{array}$ & $\begin{array}{l}\text { Instrumento de } \\
\text { recogida de datos }\end{array}$ & $\begin{array}{l}\text { Análisis de } \\
\text { datos }\end{array}$ & Objetivo & Resultados \\
\hline $\begin{array}{l}\text { Escudero et } \\
\text { al, 2010, } \\
\text { España }\end{array}$ & $\begin{array}{l}\text { Estudio } \\
\text { cualitativo con } \\
\text { tres grupos } \\
\text { focales }\end{array}$ & $\begin{array}{l}20 \text { mujeres } \\
\text { y } 1 \text { hombre }\end{array}$ & $\begin{array}{l}\text { Entrevista grupal } \\
\text { semi-estructurada }\end{array}$ & $\begin{array}{lr}\text { Análisis } & \text { de } \\
\text { contenido } & \text { con } \\
\text { apoyo } & \text { del } \\
\text { sistema } & \\
\text { informático } & \\
\text { Nudist VIVO } & \end{array}$ & $\begin{array}{l}\text { Conocer las } \\
\text { experiencias y } \\
\text { expectativas } \\
\text { de las } \\
\text { personas con } \\
\text { fibromialgia }\end{array}$ & 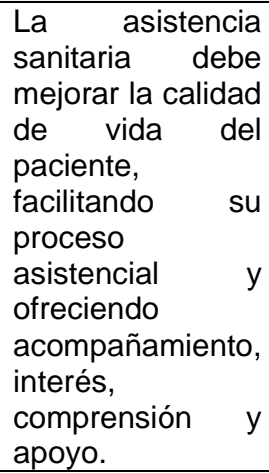 \\
\hline $\begin{array}{l}\text { Kengen } \\
\text { Traska et al, } \\
2012 \text {, } \\
\text { E.E.U.U. }\end{array}$ & $\begin{array}{l}\text { Estudio } \\
\text { cualitativo } \\
\text { descriptivo }\end{array}$ & 8 mujeres & $\begin{array}{l}\text { Entrevista grupal } \\
\text { con preguntas } \\
\text { abiertas }\end{array}$ & $\begin{array}{l}\text { Análisis } \\
\text { contenido }\end{array}$ & $\begin{array}{l}\text { Determinar } \\
\text { que } \\
\text { estrategias } \\
\text { desarrolla una } \\
\text { persona con } \\
\text { fibromialgia } \\
\text { para convivir } \\
\text { con sus } \\
\text { síntomas }\end{array}$ & $\begin{array}{l}\text { Las personas } \\
\text { que padecen } \\
\text { fibromialgia } \\
\text { desarrollan } \\
\text { estrategias que } \\
\text { les permiten vivir } \\
\text { frente a un dolor } \\
\text { crónico y fatiga. } \\
\text { Incluyendo } \\
\text { técnicas de } \\
\text { distracción, } \\
\text { estimulación, } \\
\text { planificación con } \\
\text { apoyo social y } \\
\text { familiar. }\end{array}$ \\
\hline $\begin{array}{l}\text { Arnold et al, } \\
\text { 2008, } \\
\text { E.E.U.U. }\end{array}$ & $\begin{array}{l}\text { Estudio } \\
\text { cualitativo }\end{array}$ & 48 mujeres & $\begin{array}{l}\text { Entrevista semi- } \\
\text { estructurada en } \\
\text { seis sesiones de } \\
\text { grupos focales }\end{array}$ & $\begin{array}{l}\text { Proceso de } \\
\text { codificación- } \\
\text { categorización } \\
\text { basado en } \\
\text { Strauss y } \\
\text { Corbin }\end{array}$ & $\begin{array}{l}\text { Evaluar el } \\
\text { impacto de la } \\
\text { fibromialgia en } \\
\text { la calidad de } \\
\text { vida de los } \\
\text { pacientes }\end{array}$ & 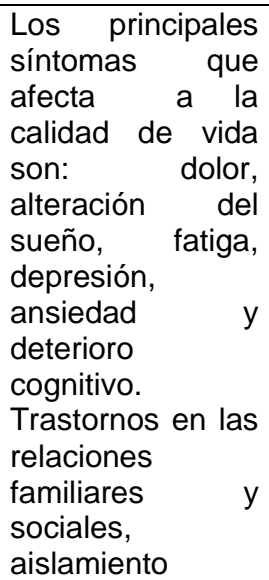 \\
\hline
\end{tabular}




\begin{tabular}{|c|c|c|c|c|c|c|}
\hline & & & & & & $\begin{array}{l}\text { social, reducción } \\
\text { de la actividad } \\
\text { física y pérdida } \\
\text { de la carrera } \\
\text { profesional. }\end{array}$ \\
\hline $\begin{array}{l}\text { Underland y } \\
\text { Malterud, } \\
2007 \text {, } \\
\text { Noruega }\end{array}$ & $\begin{array}{l}\text { Estudio } \\
\text { cualitativo con } \\
\text { dos grupos } \\
\text { focales }\end{array}$ & 11 mujeres & $\begin{array}{l}\text { Entrevistas } \\
\text { semiestructuradas } \\
\text { en cuatro } \\
\text { sesiones con los } \\
\text { grupos focales }\end{array}$ & $\begin{array}{ll}\text { Codificación en } & \text { en } \\
\text { unidades de } \\
\text { significado }\end{array}$ & $\begin{array}{l}\text { Explorar las } \\
\text { experiencias y } \\
\text { consecuencias } \\
\text { del proceso de } \\
\text { diagnóstico } \\
\text { FM }\end{array}$ & $\begin{array}{l}\text { El diagnóstico } \\
\text { legitima los } \\
\text { síntomas como } \\
\text { una enfermedad } \\
\text { lo que repercute } \\
\text { en una respuesta } \\
\text { de alivio para los } \\
\text { pacientes y un } \\
\text { camino en la } \\
\text { resolución de sus } \\
\text { problemas. }\end{array}$ \\
\hline $\begin{array}{l}\text { Lempp et al, } \\
\text { 2009, Reino } \\
\text { Unido }\end{array}$ & $\begin{array}{l}\text { Estudio } \\
\text { cualitativo }\end{array}$ & $\begin{array}{l}12 \\
\text { pacientes }\end{array}$ & $\begin{array}{l}\text { Entrevista semi- } \\
\text { estructurada }\end{array}$ & $\begin{array}{l}\text { Proceso de } \\
\text { codificación- } \\
\text { categorización }\end{array}$ & $\begin{array}{l}\text { Entender las } \\
\text { experiencias } \\
\text { de vivir con } \\
\text { esta } \\
\text { enfermedad a } \\
\text { largo plazo. }\end{array}$ & $\begin{array}{l}\text { La fibromialgia es } \\
\text { una enfermedad } \\
\text { que se entiende } \\
\text { poco y que } \\
\text { demanda } \\
\text { grandes recursos } \\
\text { sanitarios. Hay } \\
\text { que prestar } \\
\text { mayor atención a } \\
\text { los vínculos entre } \\
\text { los modelos } \\
\text { explicativos de } \\
\text { los pacientes y el } \\
\text { personal y a la } \\
\text { interrelación } \\
\text { entre las } \\
\text { complejas } \\
\text { necesidades } \\
\text { físicas, } \\
\text { psicológicas y } \\
\text { sociales. }\end{array}$ \\
\hline $\begin{array}{l}\text { Hamnes et } \\
\text { al, 2011, } \\
\text { Noruega }\end{array}$ & $\begin{array}{l}\text { Estudio } \\
\text { cualitativo }\end{array}$ & $\begin{array}{l}8 \text { pacientes } \\
\text { con } \\
\text { fibromialgia } \\
\text { y } 8 \\
\text { pacientes } \\
\text { con artritis } \\
\text { reumatoide }\end{array}$ & $\begin{array}{l}\text { Entrevista semi- } \\
\text { estructurada }\end{array}$ & $\begin{array}{l}\text { Análisis } \\
\text { temático }\end{array}$ & $\begin{array}{l}\text { Determinar las } \\
\text { expectativas } \\
\text { de un paciente } \\
\text { hospitalizado } \\
\text { durante una } \\
\text { semana ante } \\
\text { un programa } \\
\text { de autocuidado }\end{array}$ & $\begin{array}{l}\text { La identificación } \\
\text { de las } \\
\text { expectativas } \\
\text { previas de un } \\
\text { paciente ante un } \\
\text { programa de } \\
\text { auto-cuidado } \\
\text { ofrece importante } \\
\text { información que } \\
\text { tiene } \\
\text { implicaciones } \\
\text { para la ejecución } \\
\text { del programa. } \\
\text { Otros temas } \\
\text { como la } \\
\text { aceptación de la } \\
\text { enfermedad y la } \\
\text { gestión del } \\
\text { trabajo deben } \\
\text { incluirse en el } \\
\text { programa. }\end{array}$ \\
\hline $\begin{array}{l}\text { Steihaug, } \\
2007, \\
\text { Noruega }\end{array}$ & $\begin{array}{l}\text { Estudio } \\
\text { cualitativo }\end{array}$ & 8 mujeres & $\begin{array}{l}\text { Entrevista semi- } \\
\text { estructurada }\end{array}$ & $\begin{array}{l}\text { Condensación } \\
\text { de texto } \\
\text { sistemático } \\
\text { según el } \\
\text { Método Giorgi }\end{array}$ & $\begin{array}{l}\text { Explorar si la } \\
\text { participación } \\
\text { en un grupo } \\
\text { de tratamiento } \\
\text { para mujeres } \\
\text { con un dolor } \\
\text { crónico puede } \\
\text { ayudar a } \\
\text { desarrollar } \\
\text { estrategias }\end{array}$ & $\begin{array}{l}\text { Los participantes } \\
\text { desarrollaron } \\
\text { estrategias para } \\
\text { el manejo de su } \\
\text { dolor muscular } \\
\text { crónico. Les } \\
\text { proporciona } \\
\text { mayor seguridad } \\
\text { en sí mismos que } \\
\text { ayudan a } \\
\text { relacionarse con }\end{array}$ \\
\hline
\end{tabular}




\begin{tabular}{|c|c|c|c|c|c|c|}
\hline & & & & & $\begin{array}{l}\text { para manejar } \\
\text { su dolor. }\end{array}$ & $\begin{array}{l}\text { su entorno y } \\
\text { contribuye a la } \\
\text { promoción de la } \\
\text { comprensión de } \\
\text { la enfermedad. }\end{array}$ \\
\hline $\begin{array}{l}\text { LaChapelle } \\
\text { et al, 2008, } \\
\text { Canadá }\end{array}$ & $\begin{array}{l}\text { Estudio } \\
\text { cualitativo }\end{array}$ & 45 mujeres & $\begin{array}{l}\text { Once grupos } \\
\text { focales. Notas de } \\
\text { campo }\end{array}$ & $\begin{array}{l}\text { Análisis } \\
\text { temático según } \\
\text { el Método de } \\
\text { Braun y Clarke }\end{array}$ & $\begin{array}{l}\text { Explorar las } \\
\text { definiciones } \\
\text { personales de } \\
\text { aceptación y } \\
\text { los factores } \\
\text { que facilitan o } \\
\text { dificultan la } \\
\text { aceptación del } \\
\text { dolor }\end{array}$ & 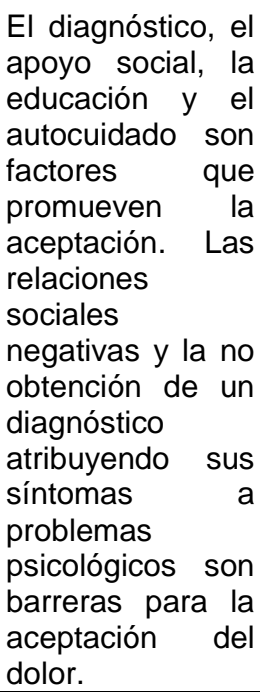 \\
\hline $\begin{array}{l}\text { Wuytack y } \\
\text { Miller, 2011, }\end{array}$ & $\begin{array}{l}\text { Estudio } \\
\text { cualitativo } \\
\text { empleando } \\
\text { fenomenología } \\
\text { descriptiva }\end{array}$ & 6 mujeres & $\begin{array}{l}\text { Entrevistas semi- } \\
\text { estructuradas }\end{array}$ & $\begin{array}{l}\text { Proceso de } \\
\text { codificación- } \\
\text { categorización }\end{array}$ & $\begin{array}{l}\text { Lograr una } \\
\text { mejor } \\
\text { comprensión } \\
\text { de la } \\
\text { experiencia } \\
\text { subjetiva de la } \\
\text { fibromialgia } \\
\text { centrándose } \\
\text { en el impacto } \\
\text { personal, } \\
\text { laboral y social }\end{array}$ & $\begin{array}{l}\text { Surgieron cuatro } \\
\text { temas principales } \\
\text { que afectan a la } \\
\text { calidad de vida } \\
\text { de } \\
\text { pacientes: estos } \\
\text { impacto en la } \\
\text { vida laboral, } \\
\text { personal, sobre } \\
\text { su futuro y la } \\
\text { interacción social. } \\
\text { La necesidad de } \\
\text { un proceso de } \\
\text { diagnóstico más } \\
\text { eficaz y una } \\
\text { mayor educación } \\
\text { sobre la } \\
\text { fibromialgia } \\
\text { implica a los } \\
\text { profesionales } \\
\text { sanitarios. }\end{array}$ \\
\hline $\begin{array}{l}\text { CunningHam } \\
\text { y Jillings, } \\
2006 \text {, } \\
\text { Canadá }\end{array}$ & $\begin{array}{l}\text { Estudio } \\
\text { cualitativo con } \\
\text { diseño } \\
\text { descriptivo } \\
\text { interpretativo }\end{array}$ & $\begin{array}{l}7 \text { mujeres y } \\
1 \text { hombre }\end{array}$ & $\begin{array}{l}\text { Entrevistas semi- } \\
\text { estructuradas }\end{array}$ & $\begin{array}{l}\text { Método } \\
\text { analítico } \\
\text { comparativo }\end{array}$ & $\begin{array}{l}\text { La experiencia } \\
\text { de vivir con } \\
\text { FM, identificar } \\
\text { las políticas } \\
\text { sociales y los } \\
\text { problemas de } \\
\text { salud que les } \\
\text { afectan } \\
\text { principalmente }\end{array}$ & $\begin{array}{lr}\text { Vivir con } & \text { FM } \\
\text { consiste } & \text { en } \\
\text { experimentar } & \text { y } \\
\text { gestionar } & \text { una } \\
\text { gran cantidad de } \\
\text { síntomas cuya } \\
\text { invisibilidad y la } \\
\text { falta } & \text { de } \\
\text { previsibilidad } & \\
\text { supone } & \text { un } \\
\text { importante } & \\
\text { impacto en la } \\
\text { calidad de vida } \\
\text { de los pacientes } \\
\text { a todos } \\
\text { niveles. los } \\
\text { Las personas con } \\
\text { FM r se } \\
\text { beneficiarán r si } \\
\text { hay } & \text { más } \\
\text { profesionales }\end{array}$ \\
\hline
\end{tabular}




\begin{tabular}{|c|c|c|c|c|c|c|}
\hline & & & & & & $\begin{array}{lr} & \text { sanitarios, } \\
\text { como familiares y } \\
\text { amigos que les } \\
\text { ofrezcan } \\
\text { mejor apoyo. }\end{array}$ \\
\hline $\begin{array}{l}\text { Martín et al, } \\
2009 \text {, } \\
\text { E.E.U.U. }\end{array}$ & $\begin{array}{l}\text { Estudio } \\
\text { cualitativo }\end{array}$ & $\begin{array}{l}16 \text { mujeres } \\
\text { y } 4 \\
\text { hombres }\end{array}$ & $\begin{array}{ll}\text { Entrevistas } & \text { con } \\
\text { una } & \text { guía } \\
\text { estructurada } & \end{array}$ & $\begin{array}{l}\text { Análisis de } \\
\text { contenido }\end{array}$ & $\begin{array}{l}\text { Evaluar el } \\
\text { impacto de la } \\
\text { FM en los } \\
\text { pacientes } \\
\text { incluyendo el } \\
\text { sueño }\end{array}$ & $\begin{array}{l}\text { Los pacientes } \\
\text { reforzaron } \\
\text { consistentemente } \\
\text { que la FM tiene } \\
\text { un impacto } \\
\text { debilitante en sus } \\
\text { vidas } \\
\text { especialmente } \\
\text { para conciliar el } \\
\text { sueño } \\
\text { permanecer y } \\
\text { dormido. }\end{array}$ \\
\hline $\begin{array}{l}\text { Humphrey et } \\
\text { al, 2010, } \\
\text { Reino Unido }\end{array}$ & $\begin{array}{l}\text { Estudio } \\
\text { cualitativo }\end{array}$ & $\begin{array}{l}28 \text { mujeres } \\
\text { y } 12 \\
\text { hombres }\end{array}$ & $\begin{array}{l}\text { Entrevistas } \\
\text { abiertas }\end{array}$ & $\begin{array}{l}\text { Proceso de } \\
\text { codificación- } \\
\text { categorización }\end{array}$ & $\begin{array}{l}\text { Entender } \\
\text { mejor los } \\
\text { aspectos de la } \\
\text { fatiga que } \\
\text { podrían ser } \\
\text { exclusivos de } \\
\text { FM, así como } \\
\text { el impacto que } \\
\text { tiene sobre la } \\
\text { vida de los } \\
\text { pacientes }\end{array}$ & $\begin{array}{l}\text { Los participantes } \\
\text { describen la } \\
\text { fatiga como una } \\
\text { abrumadora } \\
\text { sensación de } \\
\text { cansancio que no } \\
\text { se alivia con el } \\
\text { descanso y no es } \\
\text { proporcional al } \\
\text { esfuerzo } \\
\text { realizado } \\
\text { asociado a un } \\
\text { sentimiento de } \\
\text { debilidad que } \\
\text { interfiere con la } \\
\text { motivación, las } \\
\text { actividades de la } \\
\text { vida diaria, } \\
\text { prolonga las } \\
\text { tareas y hace que } \\
\text { sea difícil } \\
\text { concentrarse o } \\
\text { recordar cosas. }\end{array}$ \\
\hline $\begin{array}{l}\text { Mannerkorpi } \\
\text { y } \quad \text { Gard, } \\
2012, \text { Suecia }\end{array}$ & $\begin{array}{l}\text { Estudio } \\
\text { cualitativo }\end{array}$ & 27 mujeres & $\begin{array}{l}\text { Cinco entrevistas } \\
\text { de grupo focales }\end{array}$ & $\begin{array}{l}\text { Análisis de } \\
\text { contenido }\end{array}$ & $\begin{array}{l}\text { Investigar } \\
\text { cuáles son los } \\
\text { problemas de } \\
\text { salud y las } \\
\text { dificultades } \\
\text { relacionadas } \\
\text { con el trabajo } \\
\text { y la FM }\end{array}$ & $\begin{array}{l}\text { Se identificaron } \\
\text { los problemas de } \\
\text { salud que } \\
\text { interfieren en las } \\
\text { demandas } \\
\text { físicas, } \\
\text { psicosociales y } \\
\text { organización en } \\
\text { el trabajo: la } \\
\text { limitada } \\
\text { capacidad física, } \\
\text { aumento del } \\
\text { estrés y una } \\
\text { mayor necesidad } \\
\text { de descanso. }\end{array}$ \\
\hline $\begin{array}{l}\text { Bieber et al, } \\
2006, \\
\text { Alemania }\end{array}$ & $\begin{array}{l}\text { Combinación } \\
\text { de un estudio } \\
\text { cualitativo y } \\
\text { enfoque } \\
\text { cuantitativo }\end{array}$ & $\begin{array}{l}67 \\
\text { pacientes }\end{array}$ & $\begin{array}{l}\text { Estudio } \\
\text { cualitativo: } \\
\text { entrevistas semi- } \\
\text { estructuradas } \\
\text { Enfoque } \\
\text { cuantitativo: } \\
\text { Completar } \\
\text { cuestionario en la } \\
\text { consulta inicial, a } \\
\text { los } 3 \text { meses y al } \\
\text { año }\end{array}$ & $\begin{array}{l}\text { Estudio } \\
\text { cualitativo: } \\
\text { proceso de } \\
\text { codificación- } \\
\text { codificación. } \\
\text { Enfoque } \\
\text { cuantitavivo: } \\
\text { programa de } \\
\text { codificación } \\
\text { ANOVA }\end{array}$ & $\begin{array}{l}\text { Se } \\
\text { investigaron } \\
\text { los efectos de } \\
\text { la toma de } \\
\text { decisiones } \\
\text { compartidas } \\
\text { en la } \\
\text { interacción } \\
\text { médico- } \\
\text { paciente }\end{array}$ & $\begin{array}{l}\text { El tratamiento de } \\
\text { acuerdo con la } \\
\text { toma } \\
\text { decisiones } \\
\text { compartidas lleva } \\
\text { a una mejor } \\
\text { relación médico- } \\
\text { paciente desde la } \\
\text { perspectiva de } \\
\text { los pacientes y } \\
\text { también de los } \\
\text { médicos. Mejora } \\
\text { el afrontamiento }\end{array}$ \\
\hline
\end{tabular}




\begin{tabular}{|c|c|c|c|c|c|c|}
\hline & & & & & & $\begin{array}{l}\text { en pacientes con } \\
\text { FM y fomenta a } \\
\text { adoptar planes } \\
\text { de tratamiento } \\
\text { más activos. }\end{array}$ \\
\hline $\begin{array}{l}\text { Rodham et } \\
\text { al, } 2010 \text {, } \\
\text { Reino Unido }\end{array}$ & $\begin{array}{l}\text { Estudio } \\
\text { cualitativo }\end{array}$ & $\begin{array}{l}4 \text { mujeres } \\
\text { con FM y } \\
\text { sus } \\
\text { cuidadores } \\
\text { conyugales }\end{array}$ & $\begin{array}{l}\text { Entrevistas semi- } \\
\text { estructuradas }\end{array}$ & $\begin{array}{l}\text { Análisis } \\
\text { fenomenológico } \\
\text { interpretativo } \\
\text { con el enfoque } \\
\text { de Smith y } \\
\text { Osborn }\end{array}$ & $\begin{array}{l}\text { Explorar las } \\
\text { experiencias } \\
\text { tanto de las } \\
\text { personas que } \\
\text { sufren FM } \\
\text { como de sus } \\
\text { cuidadores } \\
\text { conyugales }\end{array}$ & 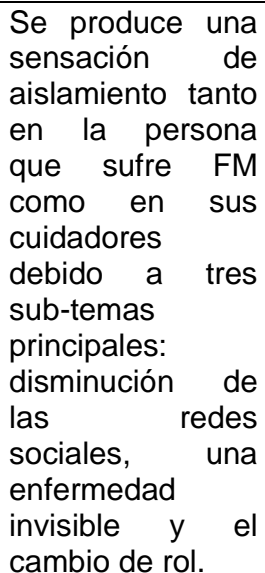 \\
\hline
\end{tabular}

\section{DISCUSIÓN}

La metodología cualitativa es idónea para profundizar en la experiencia de los pacientes ya que permiten acceder a informaciones difíciles de alcanzar a través de otras metodologías y ahondar en la comprensión de fenómenos complejos. Las técnicas de investigación cualitativas dan voz y carácter de sujetos activos a quienes participan en estos estudios ${ }^{(1,9,15)}$.

Esto es esencial en el caso de las personas con fibromialgia al ser una enfermedad por una parte poco conocida y analizada desde el punto de vista de sus consecuencias sociales y emocionales y por otra parte una enfermedad con profundas implicaciones psíquicas, sociales y laborales ${ }^{(5,11,26,32)}$.

En la presente revisión de la literatura es necesario reconocer las limitaciones en su planteamiento tanto como en los resultados obtenidos. En primer lugar se encuentra indudablemente el sesgo de selección ya que se han recogido textos de corte cualitativo donde se trata principalmente el tema a estudio desde la perspectiva subjetiva de la propia persona, su experiencia vivida pero es precisamente esta faceta de la enfermedad la que se pretende explorar.

Por otro lado, en relación a la estrategia seguida para la localización de los artículos igualmente se ve afectada por esta limitación puesto que en el momento en el que se seleccionaron los textos que se han creído más relevantes los criterios fueron muy explícitos en cuanto a los que resultaban interesantes donde se exploraba el punto de vista de la persona enferma y la experiencia vivida individualmente.

Al seleccionar los artículos se ha seguido el principio más específico en investigación cualitativa que es la saturación de la información donde se ha observado una mayor cantidad de artículos de procedencia anglosajona y estadounidense ello se percibe en la evidencia de la escasa investigación donde el enfermo es el protagonista en la actualidad.

La revisión de la bibliografía publicada respecto a la fibromialgia evidencia un aumento de los estudios de tipo fenomenológico o cualitativo en los últimos 10 años, 
suponemos que es para dar una respuesta a la demanda de estos pacientes respecto a su trato por parte del sistema sanitario, con lo cual se hace necesario un giro transcendental en la forma en la que se ha enfocado la enfermedad que provocará que se tenga en cuenta la opinión de estas personas respecto a la manera en la que se establece su plan de cuidados por parte de cualquier profesional sanitario.

Los resultados ponen de manifiesto la falta de conocimiento de los profesionales sanitarios acerca del abordaje de la enfermedad ante los primeros síntomas y la necesidad de un rápido diagnóstico. La necesidad de un diagnóstico precoz es el principal punto de partida para el tratamiento y abordaje de la enfermedad ya que de ello depende la calidad de vida de la persona y el impacto de la FM tiene en las distintas esferas a las que afecta.

\section{CONCLUSIONES}

Este trabajo revela que la fibromialgia tiene un profundo impacto en la vida de los pacientes. Los síntomas más consistentemente nombrados son el dolor, la fatiga, trastornos del sueño, depresión, ansiedad y alteraciones cognitivas. Afecta considerablemente a la calidad de vida y a la función social y profesional.

Los pacientes reconocen que hay una falta de compresión y de aceptación social de la fibromialgia, se producen sentimientos de frustración en la interacción con los demás, sentimientos que son provocados por la invisibilidad de su enfermedad y la dificultad de explicar su situación.

Las personas no son tomadas en serio por sus amigos y familiares ya que no hay evidencia de enfermedad debido a que se desconoce su causa y no hay ninguna prueba médica que pueda confirmarla; como consecuencia viven un período de incertidumbre que se asocia a trastornos emocionales, pero el punto de inflexión es la necesidad de un diagnóstico firme que disminuirá la ansiedad y desarrollará estrategias de afrontamiento en el paciente en este proceso de salud-enfermedad.

El objetivo principal del tratamiento es ayudar al paciente a convivir con su enfermedad, la aceptación del dolor se asocia con menores niveles de angustia, mejor funcionamiento físico, emocional y social. Además del tratamiento oficial existe evidencia de la mejoría con terapias alternativas.

La FM es más que una condición dolorosa ya que se asocia a múltiples síntomas que tienen un impacto sustancialmente negativo en la función y la calidad de vida. Entre ellas se encuentra la fatiga y las alteraciones del sueño.

La fatiga se describe normalmente por los pacientes como un cansancio físico que repercute en un mayor esfuerzo en las tareas físicas que disminuye su resistencia mental y una lentitud en el pensamiento.

Las alteraciones del sueño interfieren en las funciones diarias y en el estado de ánimo que influirá en sus relaciones sociales y tareas profesionales deseando una mejora en su capacidad para dormir indicando que tanto la fatiga y el dolor se relacionan directamente con la calidad del sueño.

El deterioro cognitivo asociado a esta enfermedad influye en la capacidad de concentrarse en las tareas o en expresarse con claridad. 
Las dificultades para seguir trabajando se relacionan a la incapacidad física, el agotamiento y a los trastornos cognitivos, no se pueden realizar ciertos trabajos que requieran movimientos repetitivos o una mayor concentración que conlleva a una disminución de horarios o cambios de puestos profesionales lo que repercute negativamente en su economía.

La incertidumbre con respecto a la evolución de a su enfermedad a lo largo del tiempo contribuye a un aumento de la ansiedad. Los pacientes también experimentan una gran cantidad de estrés debido a un camino a menudo difícil hasta el diagnóstico, tratamiento, problemas y síntomas intensos dando lugar a una carga en su salud mental que les puede conducir a trastornos emocionales.

Los pacientes demandan de los profesionales sanitarios empatía, interés, pero sobre todo información sobre el proceso, pronóstico y terapias de su enfermedad percibiendo falta de tiempo en la consulta que no cubre sus necesidades limitándose a la prescripción de fármacos, demandando servicios más ágiles y accesibles durante su seguimiento. Las intervenciones educativas y actividades orientadas al autocuidado mejoran la calidad de vida de los enfermos.

La compleja vivencia de la fibromialgia desde su inicio afecta a las diferentes esferas de la persona tanto familiar, social y laboral. En un principio considerados enfermos psiquiátricos, considerando sus síntomas como somáticos, lo que ocasiona una incertidumbre hasta que consiguen un diagnóstico firme. A partir de aquí se ven interrumpidas sus actividades cotidianas por lo que la persona lo que más necesita es un apoyo social percibido por parte de la familia, amigos y profesionales de la salud.

En la búsqueda de alternativas e información, algunas personas recurren a las asociaciones de pacientes, el apoyo de otros pacientes con fibromialgia parece ser un factor importante para liberarlos de la sensación de aislamiento. Las asociaciones proporcionan información, apoyo, refuerzo, entretenimiento, y en ocasiones terapias alternativas, aspectos que los pacientes valoran considerablemente.

La fibromialgia es una enfermedad crónica que no tiene cura. Una comprensión profunda de la experiencia de la enfermedad es por lo tanto clave en el cuidado paliativo de pacientes con esta condición. El conocimiento del significado y la realidad de vivir con esta enfermedad para el paciente podría ser considerado esencial para cualquier profesional sanitario incluyendo la necesidad de un proceso de diagnóstico eficaz y una mayor educación acerca de la experiencia de la fibromialgia.

Es importante que los profesionales de la salud sepan reconocer y validar la incertidumbre de la experiencia de la enfermedad en la fibromialgia.

La calidad percibida de la atención como la falta de contacto con las enfermeras y las actitudes de los especialistas, la falta de información y la interrelación entre las complejas necesidades físicas, psicológicas y sociales de los pacientes tomando un enfoque integral e individualizado puede mejorar los síntomas y su calidad de vida. La falta de tiempo de consulta de los médicos para los pacientes podría complementarse con consultas de enfermería.

La utilidad de los hallazgos encontrados en esta revisión es de gran importancia pues nos van a proporcionar el enfoque necesario de la situación actual en la que se 
encuentra la FM. Se hace por tanto necesario que se plantee una serie de cambios en el trato a estos pacientes desde el primer momento que acuden a los profesionales sanitarios en cuanto al diagnóstico, tratamiento y abordaje de esta enfermedad donde se pueda apreciar la perspectiva de la persona para conseguir los mejores resultados en su proceso salud-enfermedad.

Para conseguir este objetivo es necesario proporcionar mayor información al profesional sanitario acerca de la FM ya que existen importantes vacíos en sus conocimientos. En especial, en este terreno se incluye a Enfermería porque tiene un campo de actuación con innumerables posibilidades, ya que en la mayoría de los casos se encontrarán con pacientes en Atención Primaria de Salud donde deberán elaborar un plan de cuidados individualizado y holista para cada paciente, teniendo especial atención en la forma en la que vive la enfermedad, siempre diferente de un paciente a otro, y el desarrollo de sus mecanismos de afrontamiento para convivir con ella el resto de su vida.

Deben informar sobre las diferentes formas de abordar la enfermedad en cuanto a tratamientos farmacológicos, al igual que no farmacológicos, donde existe evidencia de la mejora en la calidad de vida. La promoción de la salud atañe exclusivamente a este colectivo que debe contar con las estrategias suficientes para informar de las peculiaridades de la enfermedad, incluyendo especialmente la forma en la que se debe tratar a estos pacientes con empatía y situándose en un paradigma sociocrítico en el que se crea una relación en la que se tiene muy en cuenta la opinión de la persona con respecto a su enfermedad para elaborar conjuntamente las diferentes estrategias, estableciéndose un diálogo recíproco entre profesional sanitario-paciente. Teniendo en cuenta estos resultados desde la perspectiva de los profesionales sanitarios en las estrategias de afrontamiento de pacientes con fibromialga se debe prestar atención a la importancia del apoyo familiar con la integración de programas educativos sobre el síndrome de fibromialgia para las familias de los pacientes.

\section{BIBLIOGRAFÍA}

1. Escudero Carretero MJ, García Toyos N, Prieto Rodríguez MA, Pérez Corral O, March Cerdá JC y López Doblas, M. Fibromialgia: Percepción de pacientes sobre su enfermedad y el sistema de salud. Estudio de investigación cualitativa. Reumatol Clínic [revista en internet]. 2010 [consultado el 6 mayo de 2012]; 6(1):16-22. Disponible en: http://www.ncbi.nlm.nih.gov/pubmed/21794673

2. Hidalgo F J. Fibromialgia. Consideraciones Etiopatogénicas. Rev Soc Esp Dolor [revista en internet]. 2011 [consultado el 29 mayo de 2012]; 18(6):342-350 Disponible en: http://scielo.isciii.es/scielo.php?pid=S1134-80462011000600005\&script=sci arttext 3. López Espino M, Mingote Adán JC. Fibromialgia. Clínica y Salud [revista en internet]. 2008 [consultado el 14 de mayo de 2012]; 19(3):343-358. Disponible en: http://redalyc.uaemex.mx/redalyc/pdf/1806/180617473005.pdf

4. Kengen Traska T, Rutledge DN, Mouttapa M, Weiss J, Aquino J. Strategies used for managing symptoms by women with fibromyalgia. J Clin Nurs [revista en internet]. 2012 Mar [consultado el 23 de junio de 2012]; 21(5-6):626-35. Disponible en: http://www.ncbi.nlm.nih.gov/pubmed/21323780

5. Ubago Linares MC, Ruiz Pérez I, Bermejo Pérez MJ. Características clínicas y psicosociales de personas con fibromialgia. Repercusión del diagnóstico sobre sus actividades. Rev Esp Salud Pública [revista en internet]. 2005 Nov [consultado el 12 de mayo de 2012]; 79(6):683-695. Disponible en: http://scielo.isciii.es/pdf/resp/v79n6/original5.pdf 
6. Restrepo Medraño JC, Rojas JG. (2011). Fibromialgia ¿Qué deben saber y evaluar los profesionales de enfermería? Invest Educ Enferm [revista en internet]. 2011 [consultado el 4 de mayo de 2012]; 29(2):305-314. Disponible en: http://aprendeenlinea.udea.edu.co/revistas/index.php/iee/article/view/4805/9375

7. Martínez Ferrero, P. Fibromialgia: Una nueva enfermedad.... ० una antigua conocida. Inform Psiqu [revista en internet]. 2010 [consultado el 5 de mayo de 2012]; 199:19-38.

Disponible

http://www.revistahospitalarias.org/info_2010/01_199_03.htm

8. Rodríguez Muñiz RM, Orta González MA, Amashta Nieto L. Cuidados de enfermería a los pacientes con fibromialgia. Enferm Glob [revista en internet]. 2010 [consultado el 8 de mayo de 2012];19. Disponible en: http://revistas.um.es/eglobal/article/viewFile/107211/101881

9. Tosal Herrero B. El cuerpo como excusa. El diagnóstico de la fibromialgia en una consulta de enfermería. Index de Enferm [revista en internet]. 2008 [consultado el 12 de junio de 2012]; 17 (1): 12-16. Disponible en: http://www.index-f.com/indexenfermeria/v17n1/6600.php

10. Klemet A, Häuser W, Brückle W. Principles of treatment, coordination of medical care and patient education in fibromyalgia syndrome and chronic widespread. Der Shmerz [revista en internet].2008[consultado el 9 de junio de 2012]; 22:283-94. Disponible en: http://www.ncbi.nlm.nih.gov/pubmed/18478270

11. Arnold LM, Crofford LJ, Mease PJ, Burgess SM, Palmer SC, Abetz L, Martín SA. Patient perspectives on the impacto of fibromyalgia. Patient Couns Educ [revista en internet]. 2008 [consultado el 10 de junio de 2012]; 73 (1): 114-120. Disponible en: http://www.pec-journal.com/article/S0738-3991(08)00279-6/abstract

12. Merayo Alonso LA, Cano García FJ, Rodríguez Franco L. Un acercamiento bibliométrico a la investigación en fibromialgia. Reumatol Clin [revista en internet].2007 [consultado el 6 de junio de 2012]; 3:55-62. Disponible en: http://apps.elsevier.es/watermark/ctl_servlet? $\mathrm{f}=10$ \&pident_articulo=13100436\&pident _usuario $=0 \&$ pcontactid $=\&$ pident_revista $=273 \&$ ty $=126 \&$ accion $=$ L\&origen $=$ reuma $\% 20 \&$ web=http://www.reumatologiaclinica. org\&lan=es\&fichero=273v3n2a13100436pdf001.p df

13. Undeland $M$, Malterud $K$.The fibromyalgia diagnosis: hardly helpful for the patients? A qualitative focus group study. Scand J Prim Health Care [revista en internet]. 2007 Dec.[consultado el 12 de junio de 2012]; 25(4):250-5. Disponible en: http://www.ncbi.nlm.nih.gov/pmc/articles/PMC3379768/

14. Carmona L. Revisión sistemática: ¿el diagnóstico en sí de fibromialgia tiene algún efecto deletéreo sobre el pronóstico?. Reumatol Clin [revista en internet]. 2006. [consultado el 6 de junio de 2012]; 2:52-7. Disponible en: http://apps.elsevier.es/watermark/ctl servlet? $\mathrm{f}=10$ \&pident articulo=13085114\&pident usuario $=0$ \&pcontactid $=\&$ pident revista $=273 \&$ ty $=23 \&$ accion $=$ L\&origen $=$ reuma $\% 20 \& \mathrm{~W}$ $\mathrm{eb}=\mathrm{http}: / / \mathrm{www}$.reumatologiaclinica.org\&lan=es\&fichero=273v2n2a13085114pdf001.pdf 15. Castro Pedraza ME. La fibromialgia, en el mejor momento de la vida. Index de Enferm [revista en internet]. 2007 [consultado el 6 de junio de 2012]; 16 (56). Disponible en: http://scielo.isciii.es/scielo.php?script=sci arttext\&pid=S113212962007000100012

16. Lempp HK, Hatch SL, Carville SF, Choy EH. Patient's experiences of living with and receiving treatment for fibromyalgia syndrome: a qualitative study. BMC Musculoskelet Disord [revista en internet]. 2009 [consultado el 5 de junio de 2012]; 10: 110-124. Disponible en: http://www.ncbi.nlm.nih.gov/pubmed/19811630

17. Rivera J, Alegre $\mathrm{C}$, Nishishinya MB. Evidencias terapéuticas en fibromialgia. Reumatol Clin [revista en internet].2006 [consultado el 15 de mayo de 2012]; 2:34-7. Disponible

en: 
http://apps.elsevier.es/watermark/ctl servlet? $\mathrm{f}=10$ \&pident articulo=13085663\&pident usuario $=0$ \&pcontactid $=$ \&pident revista $=273 \&$ ty $=59$ \&accion $=$ L\&origen $=$ reuma $\% 20 \& \mathrm{w}$ $\mathrm{eb}=\mathrm{http}: / / \mathrm{ww}$.reumatologiaclinica. org\&lan=es\&fichero=273v2nExtra.1a13085663pdf0 $01 . \mathrm{pdf}$

18. Hamnes B, Hauge MI, Kjeken I, Hagen KB. 'I have come here to learn how to cope with my illness, not to be cured': a qualitative study of patient expectations prior to a one-week self-management programme. Musculoskeletal Care [revista en internet]. 2011 Dec. [consultado el 4 de junio de 2012]; 9(4):200-10. Disponible en: http://www.ncbi.nlm.nih.gov/pubmed/21774066

19. Cabezas Prieto E.M. Desde el flequillo hasta el último dedo del pie. Arch Memoria [internet]. 2010 [consultado el 30 de mayo de 2012]; 7 (1). Disponible en: http://www.index-f.com/memoria/7/r11004.php

20. Sim J, Madden S. Illness experience in fibromyalgia syndrome: A metasynthesis of qualitative studies. Soc Sci Med [revista en internet]. 2008 [consultado el 20 de junio de 3012]; 67:57-67. 17. Disponible en: http://www.ncbi.nlm.nih.gov/pubmed/18423826 21. Pernia Romero A. La fibromialgia no debe ser ese cajón de sastre. Rev. Soc Esp Dolor [revista en internet]. 2010 [consultado el 10 de junio de 2012]; 17 (5): 225227. Disponible

en: http://apps.elsevier.es/watermark/ctl servlet? $\mathrm{f}=10$ \&pident articulo=13154223\&pident usuario $=0$ \&pcontactid $=\&$ pident revista $=289 \&$ ty $=48 \&$ accion $=$ L\&origen $=$ elsevier $\&$ web $=$ www.elsevier.es\&lan =es\&fichero=289v17n05a13154223pdf001.pdf

22. Steihaug S. Women's strategies for handling chronic muscle pain: A qualitative study. Scand J Prim Health Care [revista en internet]. 2007 [consultado el 26 de junio de 2012]; 25:44-8. Disponible en:

http://www.ncbi.n/m.nih.gov/pmc/articles/PMC3389453/

23. Mannerkorpi K, Nordeman L, Ericsson A, Arndorw M. Pool exercise for patients with fibromyalgia or chronic widespread pain: A randomized controlled trial and subgroup analyses. J Rehabil Med [revista en internet]. 2009 Sep [consultado el 6 de julio de 2012]; 9 (41):751-60. Disponible en: http://www.medicaljournals.se/jrm/content/?doi=10.2340/16501977-0409\&html=1

24. Menéndez González A, Fernández García P, Torres Viejo I. Aceptación del dolor crónico en pacientes con fibromialgia: Adaptación del Chronic Pain Acceptance Questionnaire (CPAQ) a una muestra española. Psicothema [revista en internet]. 2010 [consultado el 30 de mayo de 2012]; 22 (4): 997-1003. Disponible en: http://www.psicothema.com/pdf/3832.pdf

25. LaChapelle DL, Lavoie S, Boudreau BA. The meaning and process of pain acceptance. Perceptions of women living with arthritis and fibromyalgia. Pain Manag Res [revista en internet]. 2008 [consultado el 5 de junio de 2012]; 13 (3): 201-10. Disponible en: http://www.ncbi.nlm.nih.gov/pmc/articles/PMC2671308/

26. Wuytack $F$, Miller $P$. The lived experience of fibromyalgia in female patients, a phenomenological study. Chiropr Man Therap [revista en internet]. 2011 [consultado el 30 de junio de 2012]; 19: 22. Disponible en: http://www.ncbi.nlm.nih.gov/pmc/articles/PMC3197545/

27. Kool MB, Van Middendorp H, Boeije HR, Geenen R. Understanding the lack of understanding: Invalidation from the perspective of the patient with fibromyalgia. Artrithis Care \& Research [revista en internet]. 2009 [acceso 2 de Julio de 2012]; 12 (61): 1650-1656. Disponible en: http://onlinelibrary.wiley.com/doi/10.1002/art.24922/pdf

28. Cunningham MM, Jillings $C$. Individuals' descriptions of living with fibromyalgia.. Clin Nurs Res [revista en internet]. 2006 Nov [consultado el 5 de Julio de 2012]; 15(4):258-73. Disponible en: http://www.ncbi.nlm.nih.gov/pubmed/17056769 
29. Martín S, Arthi C, Zografos L, Gergana Z. Evaluation of the impact of fibromyalgia on patients' sleep and the content validity of two sleep scales. Health Qual Life Outcomes [revista en internet]. 2009 [consultado el 12 de junio de 2012]; 7: 64. Disponible en: $\quad$ http://www.ncbi.nlm.nih.gov/pmc/articles/PMC2717926/\#uincbiinpagenav-2

30. Stuifbergen AK, Phillips L, Carter P, Morrison J, Todd A. Subjective and objective sleep difficulties in women with fibromyalgia syndrome. J Am Acad Nurse Pract [revista en internet]. 2010 Oct [consultado el 9 de Julio de 2012]; 22(10):548-56. Disponible en: http://www.ncbi.nlm.nih.gov/pubmed/21040089

31. Humphrey L, Arbuckle R, Mease F, Williams DA, Dannesdiold Samsoe B, Gilbert C. 2010. Fatigue in fibromyalgia: a conceptual model informed by patient interviews. BMC Musculoskelet Disord [revista en internet]. 2010 [consultado el 8 de junio de 2012]; 20: 211-16. Disponible en: http://www.biomedcentral.com/1471-2474/11/216

31. Humphrey L, Arbuckle R, Mease F, Williams DA, Dannesdiold Samsoe B, Gilbert C. 2010. Fatigue in fibromyalgia: a conceptual model informed by patient interviews. BMC Musculoskelet Disord [revista en internet]. 2010 [consultado el 8 de junio de 2012]; 20: 211-16. Disponible en: http://www.biomedcentral.com/1471-2474/11/216

32. De Felipe García Bardona V, Castel Bernala B, Vidal Fuentesb J. Evidencia científica de los aspectos psicológicos en la fibromialgia. Posibilidades de intervención. Reumatol Clin [revista en internet]. 2006 [consultado el 17 de mayo de 2012]; 2:38-43. Disponible en: http://apps.elsevier.es/watermark/ctl servlet? $\mathrm{f}=10$ \&pident articulo=13085664\&pident usuario=0\&pcontactid=\&pident revista $=273 \& t y=60 \&$ accion $=$ L\&origen $=$ reuma $\% 20 \& \mathrm{w}$ eb=http://www.reumatologiaclinica.org\&lan=es\&fichero=273v2nExtra.1a13085664pdf0 01.pdf

33. Santiago Rojano GP, León Vilches I, Periáñez Cordero MR. Efectividad de las intervenciones de la enfermera de salud mental en mujeres con fibromialgia para un mejor afrontamiento a las actividades de la vida diaria. Biblioteca Las Casas [internet]. 2011 [consultado el 10 de mayo de 2012]; 7 (3). Disponible en: http://www.indexf.com/lascasas/documentos/lc0618.pdf

34. Mannerkorpi K, Gard G. Hinders for continued work among persons with fibromyalgia. BMC Musculoskeletal Disorders [revista en internet]. 2012 [consultado el 12 de julio de 2012]; 13:96. Disponible en: http://www.ncbi.nlm.nih.gov/pmc/articles/PMC3413509/pdf/1471-2474-13-96.pdf

35. Löfgren M, Ekholm J, Ohman A. 'A constant struggle': successful strategies of women in work despite fibromyalgia. Disabil Rehabil [revista en internet]. $2006 \mathrm{Apr}$ [consultado el 5 de julio de 2012]; 28(7):447-55. Disponible en: http://www.justbetweenyouandme.net/Therapists/Documents/SuccessfulStrategiesFibr o.pdf

36. Revuelta Evrard E, Segura Escobar E, Paulino Tevar J. Depresión, ansiedad y fibromialgia. Rev Soc Esp Dolor [revista en internet]. 2010 [consultado el 22 de junio $\begin{array}{lllll}\text { de 2012]; } & 17 & \text { (7):326-332. } & \text { Disponible }\end{array}$ http://scielo.isciii.es/pdf/dolor/v17n7/revision1.pdf

37. Bieber C, Müller KG, Blumenstiel K, Schneider A, Richter A, Wilke S, Hartmann M, Eich W. Long-term effects of a shared decision-making intervention on physicianpatient interaction and outcome in fibromyalgia. A qualitative and quantitative 1 year follow-up of a randomized controlled trial. Patient Educ Couns [revista en internet]. 2006 Nov [consultado el 9 de julio de 2012]; 63(3):357-66 Disponible en: http://www.ncbi.nlm.nih.gov/pubmed/16872795

38. Eide $\mathrm{H}$, Sibbern T, Johannessen T. Empathic accuracy of nurses' immediate responses to fibromyalgia patients' expressions of negative emotions: an evaluation using interaction analysis.. J Adv Nurs [revista en internet]. 2011 Jun [consultado el 12 
de Julio de 2012];67(6):1242-53.

en:

http://www.ncbi.nlm.nih.gov/pubmed/21306422

39. Martínez Lavín M. La relación médico-paciente en el contexto de la fibromialgia. Tribulaciones y propuestas. Reumatol Clin [revista en internet]. 2007 [consultado el 22 de mayo de 2012]; 3:53-4. Disponible en: http://apps.elsevier.es/watermark/ctl servlet? $\mathrm{f}=10$ \&pident articulo=13100435\&pident usuario $=0$ \&pcontactid $=$ \&pident revista $=273 \&$ ty $=125 \&$ accion $=$ L\&origen $=$ reuma $\% 20$ \& web=http://www. reumatologiaclinica.org\&lan=es\&fichero=273v3n2a13100435pdf001.p $\underline{\mathrm{df}}$

40. Wiles $\mathrm{R}$, Cotty $\mathrm{CH}$, Gibson BE. Hope, expectations and recovery from illness: a narrative synthesis of qualitative research. Journal of Advanced Nursing [revista en internet]. 2008 [consultado el 5 de julio de 2012]; 6 (64): 564-573. Disponible en: http://onlinelibrary.wiley.com/doi/10.1111/j.1365-2648.2008.04815.x/full

41. Arvidsson S, Bergman, Arvidsson B, Fridlund, Bengtsson tops A. Experiences of health-promoting self-care in people living with rheumatic diseases. Jounal of Advanced Nursing [revista en internet]. 2011 [consultado el 12 de julio de 2012]; 6 (67):1264-1272.Disponible en: http://onlinelibrary.wiley.com/doi/10.1111/i.13652648.2010.05585.x/full

42. Rodham K, Rance N, Blake D. A qualitative exploration of carers' and 'patients' experiences of fibromyalgia: one illness, different perspectives. Musculoskeletal Care [revista en internet]. 2010 Jun [consultado el 10 de julio de 2012]; 8 (2):68-77. Disponible en: http://www.ncbi.nlm.nih.gov/pubmed/20229608 